\title{
Total Curvature and Total Torsion of Knotted Polymers
}

\author{
Patrick Plunkett, ${ }^{\dagger}$ Michael Piatek, ${ }^{\ddagger}$ Akos Dobay, ${ }^{\S}$ John C. Kern, ${ }^{\dagger}$ Kenneth C. Millett, \\ Andrzej Stasiak,,${ }^{\perp}$ and Eric J. Rawdon*,\# \\ Department of Mathematics and Computer Science, Duquesne University, Pittsburgh, Pennsylvania \\ 15282; Department of Computer Science and Engineering, University of Washington, Seattle, \\ Washington 98195; Ludwig-Maximilians-Universität, Biozentrum, Grosshadernerstrasse 2, \\ 82152 Munich, Germany; Department of Mathematics, University of California, Santa Barbara, Santa \\ Barbara, California 93106; Faculty of Biology and Medicine, Laboratory of Ultrastructural Analysis, \\ University of Lausanne, Lausanne CH 1015, Switzerland; and Department of Mathematics, University \\ of St. Thomas, St. Paul, Minnesota 55105
}

Received December 1, 2006; Revised Manuscript Received February 7, 2007

\begin{abstract}
Previous work on radius of gyration and average crossing number has demonstrated that polymers with fixed topology show a different scaling behavior with respect to these characteristics than polymers with unrestricted topology. Using numerical simulations, we show here that the difference in the scaling behavior between polymers with restricted and unrestricted topology also applies to the total curvature and total torsion. For each knot type, the equilibrium length with respect to a given spatial characteristic is the number of edges at which the value of the characteristic is the same as the average for all polygons. This number appears to be correlated to physical properties of macromolecules, for example gel mobility as measured by the separation between distinct knot types. We also find that, on average, closed polymers require slightly more total curvature and slightly less total torsion than open polymers with the corresponding number of monomers.
\end{abstract}

\section{Introduction}

The simplest, and also the most fundamental, type of random walk used to model macromolecules is composed of freely jointed segments of equal length in which the individual segments have no thickness. This type of random walk is frequently used to model polymers at thermodynamic equilibrium under $\theta$-conditions or in melt phase where polymer segments that are not in a direct contact neither attract nor repel each other. ${ }^{1-9}$ Linear chains without excluded volume are believed to behave as linear polymers in $\theta$-conditions and have scaling exponent $v=0.5$. The same scaling behavior is observed in the case of phantom polygons where the simulated segments can freely pass through each other. However, when the simulated polygons without thickness are required to preserve their original topological knot type, one observes the scaling behavior of selfavoiding walks with scaling exponent $v=0.588 .{ }^{1-8}$ Interestingly, the scaling profiles of such characteristics as the radius of gyration or the average crossing number of random polygons forming different knot types exhibit distinguishable profiles due to the different correcting terms that depend upon the topology. $3,6,7$ This phenomenon is believed to reflect the physical behavior of cyclic polymers with different topologies under conditions where polymer segments that are not in direct contact neither attract nor repel each other. To understand better the statistical mechanics of polymers under these specific conditions, we use numerical simulations to determine the scaling profiles for the total curvature and total torsion of random closed polymers with fixed topology. The total curvature is a measure of accumulated bending of a polygon ${ }^{10,11}$ and is computed by

* Corresponding author. E-mail: ejrawdon@stthomas.edu.

$\dagger$ Duquesne University.

$\doteqdot$ University of Washington.

§ Ludwig-Maximilians-Universität.

" University of California, Santa Barbara.

$\perp$ University of Lausanne.

\# University of St. Thomas.
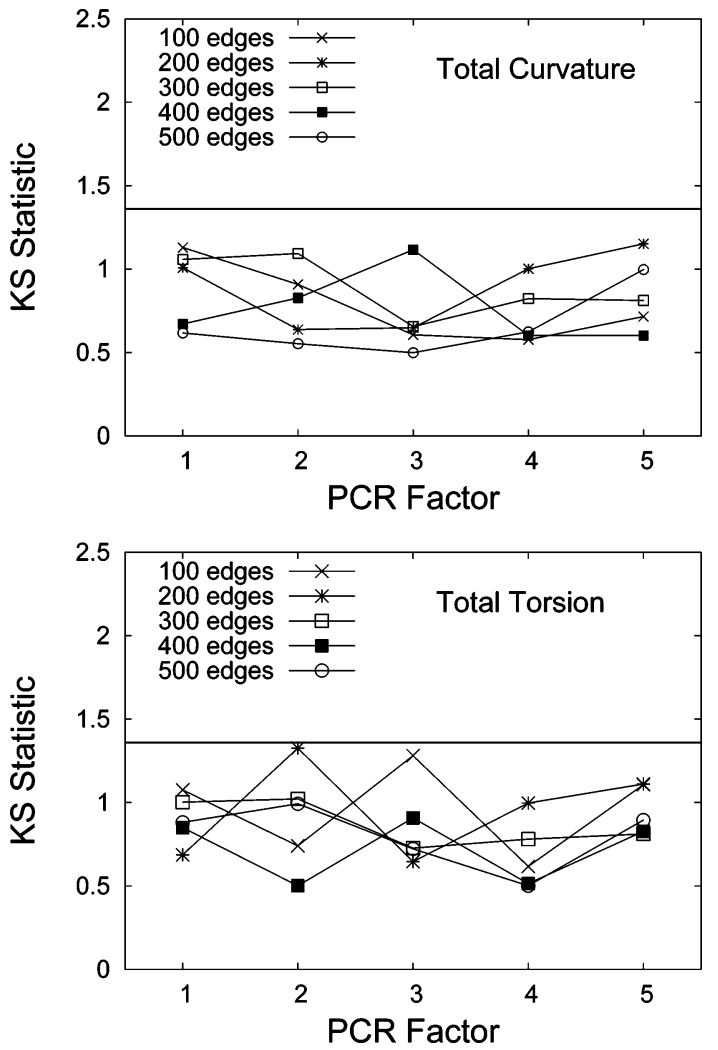

Figure 1. KS statistic values for $k n$ PCRs vs $n^{2}$ PCRs for total curvature (top) and total torsion (bottom). The $x$-axis is the factor $k$, and the $y$-axis is the KS statistic value. The five sets of data points correspond to different numbers of edges. The horizontal line is at 1.36, the statistic value which provides evidence with $95 \%$ confidence. The connecting lines are for visualization purposes only.

summing the turning angle of the edge vectors at each of the polygon's vertices. The total torsion measures the amount a polygon deviates from being planar. To compute the torsion 

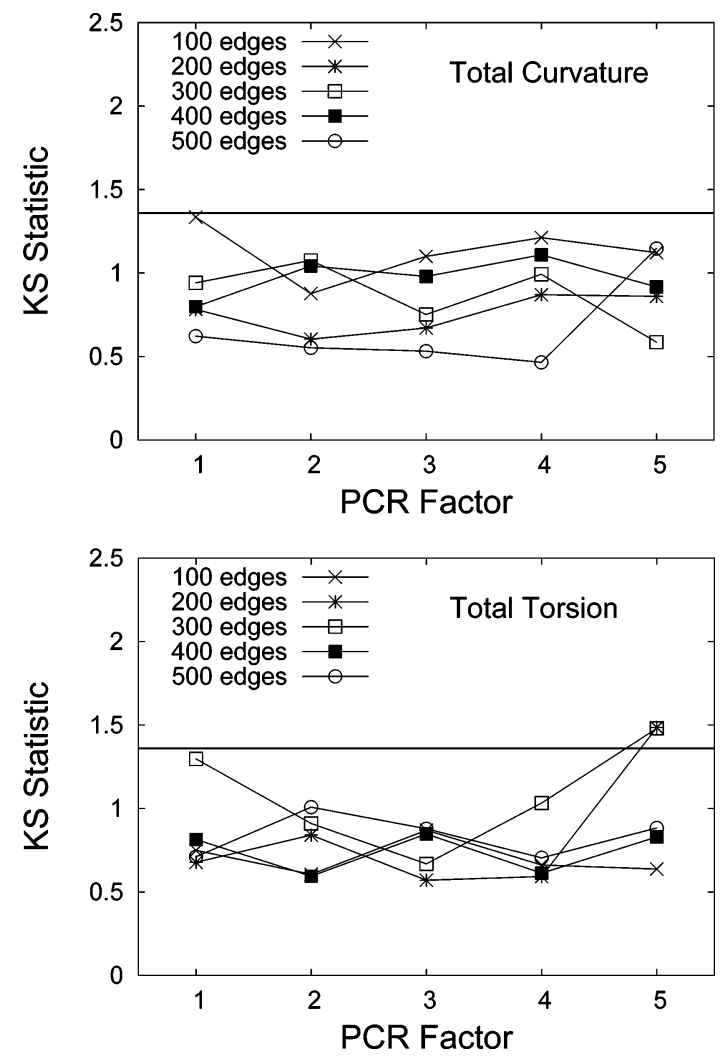

Figure 2. KS statistic values for $k n$ PCR hedgehog polygons vs $n^{2}$ crankshaft rotation Monte Carlo polygons for total curvature (top) and total torsion (bottom). The $x$-axis is the factor $k$, and the $y$-axis is the KS statistic value. The five sets of data points correspond to different numbers of edges.

angle at an edge, one determines the angle between the planes determined by the given edge and the preceding edge and by the given edge and the following edge. The total torsion is the sum of the torsion angles over the edges of the polygon.

The total curvature and total torsion provide important information about how much, and in what ways, the modeled polymers turn in space. Thus, one expects these quantities to be intimately connected with the statistical characteristics of knotting and, therefore, relevant to the properties of macromolecules. We find that the scaling profiles for each fixed nontrivial knot type intersect the scaling profiles of phantom polygons for both total curvature and total torsion. For polymers under $\theta$-conditions or in melt phase, the point of intersection defines the equilibrium length ${ }^{6,7}$ of the given knot type with respect to the given characteristic.

From a physical standpoint, the equilibrium length measures the length of polymer at which releasing the topological restraint (for a given knot type) results in no net gain or loss in the average spatial measurement (here, either total curvature or total torsion). Consider, for example, the total curvature of polymers whose knot type is that of a trefoil. When the length is shorter than the equilibrium length, releasing the topological constraint will have a tendency to result in a polymer with smaller total curvature. When the length is longer than the equilibrium length, releasing the topological constraint will have a tendency to result in a polymer with larger total curvature. The opposite is true for total torsion (Figure 4). Grosberg et al.,12,13 introduced the term overknotted and underknotted to refer to a polymer with a fixed topology and chain length that is either shorter or longer, respectively, than the equilibrium length for a given topology. In the overknotted regime (chain size below the equilibrium length) a polymer with a given knot type would have a tendency
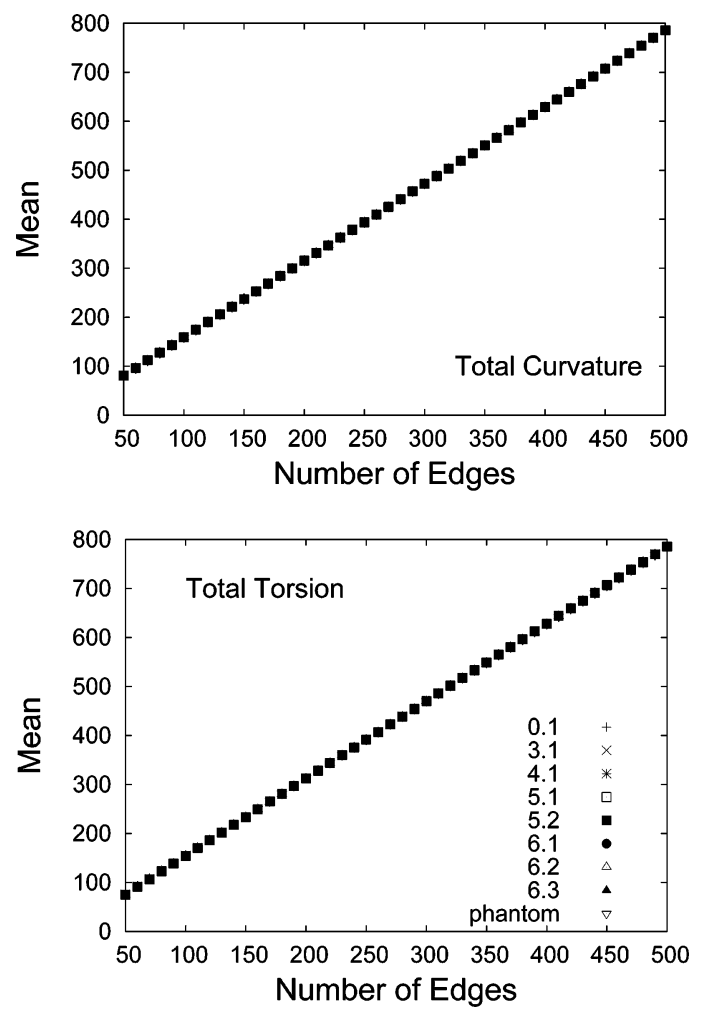

Figure 3. Scaling profiles for mean total curvature (top) and mean total torsion (bottom).
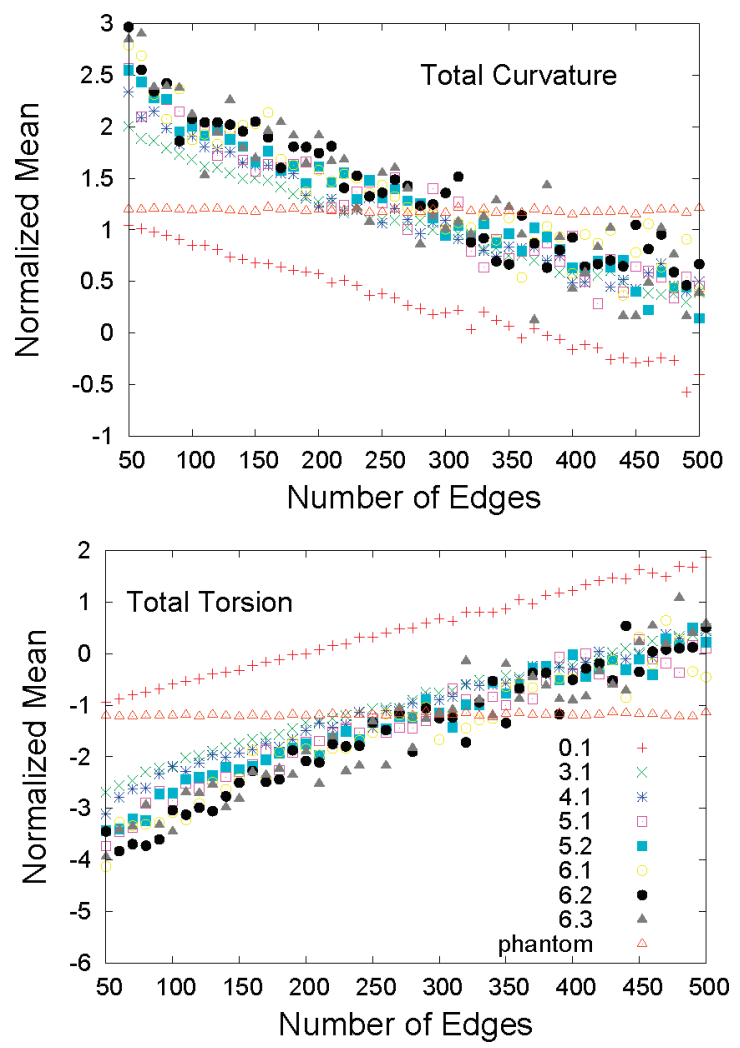

Figure 4. Scaling profiles for the normalized mean total curvature (top) and normalized mean total torsion (bottom). The phantom polygons are the nearly horizontal set of points, and the unknotted polygons are the points separated from the majority.

to simplify its knot type upon a release from a topological constraint while the opposite is the case in the underknotted regime (chain size above the corresponding equilibrium length). We will see that the equilibrium lengths with respect to total 
Table 1. Fitting Parameters for the Normalized Mean Total Curvature and Normalized Mean Total Torsion

\begin{tabular}{|c|c|c|c|c|c|c|}
\hline \multirow[b]{2}{*}{ knot } & \multicolumn{3}{|c|}{ total curvature } & \multicolumn{3}{|c|}{ total torsion } \\
\hline & $A$ & $B$ & $C$ & $A$ & $B$ & $C$ \\
\hline phantom & 1.189438 & & & -1.200496 & & \\
\hline $3_{1}$ & 0.002529 & 0.123075 & -3.64472 & -0.001439 & -0.064766 & 2.48945 \\
\hline $4_{1}$ & 0.002073 & 0.151607 & -4.08389 & 0.000664 & -0.142187 & 3.23765 \\
\hline $5_{1}$ & -0.001514 & 0.280715 & -5.46702 & 0.000371 & -0.144244 & 3.43845 \\
\hline $5_{2}$ & 0.000942 & 0.210419 & -4.95636 & -0.000587 & -0.116817 & 3.28896 \\
\hline 61 & -0.001221 & 0.291088 & -5.8159 & 0.002414 & -0.203556 & 3.94459 \\
\hline $6_{2}$ & 0.002142 & 0.212785 & -5.54977 & 0.000679 & -0.155995 & 3.69169 \\
\hline $6_{3}$ & 0.006956 & 0.059110 & -4.37179 & 0.000397 & -0.158986 & 3.77805 \\
\hline
\end{tabular}

curvature and total torsion are roughly equal for a fixed knot type. In addition, we discover that, on average, random polygons have slightly more total curvature and slightly less total torsion than random open walks. Curiously, the amounts of excess and deficit are approximately the same.

We characterize the probability profiles of prime knots with up to six crossings and determine the chain length for which the knot types reach their maximum occurrence (Figure 11). We compare these chain lengths with the total curvature and total torsion equilibrium lengths of the respective knot types. The close correspondence between the values suggests that the ratio of the entropy of a given knot to the entropy of all polygons reaches its maximum at the chain length corresponding to the equilibrium length of a given knot with respect to the total curvature or total torsion.

\section{Computations}

We analyzed equilateral random polygons ranging from 50 edges to 500 edges by a step size of 10 edges. For each number of edges, we generated 400000 random polygons using the hedgehog method ${ }^{14}$ as follows: To generate an $n$ edge (where $n$ is even) closed knot, we sample $n / 2$ edge vectors uniformly on the unit sphere. We use the GNU Scientific Libraries (GSL) function gsl_ran_dir_3d to generate the uniform vectors on the
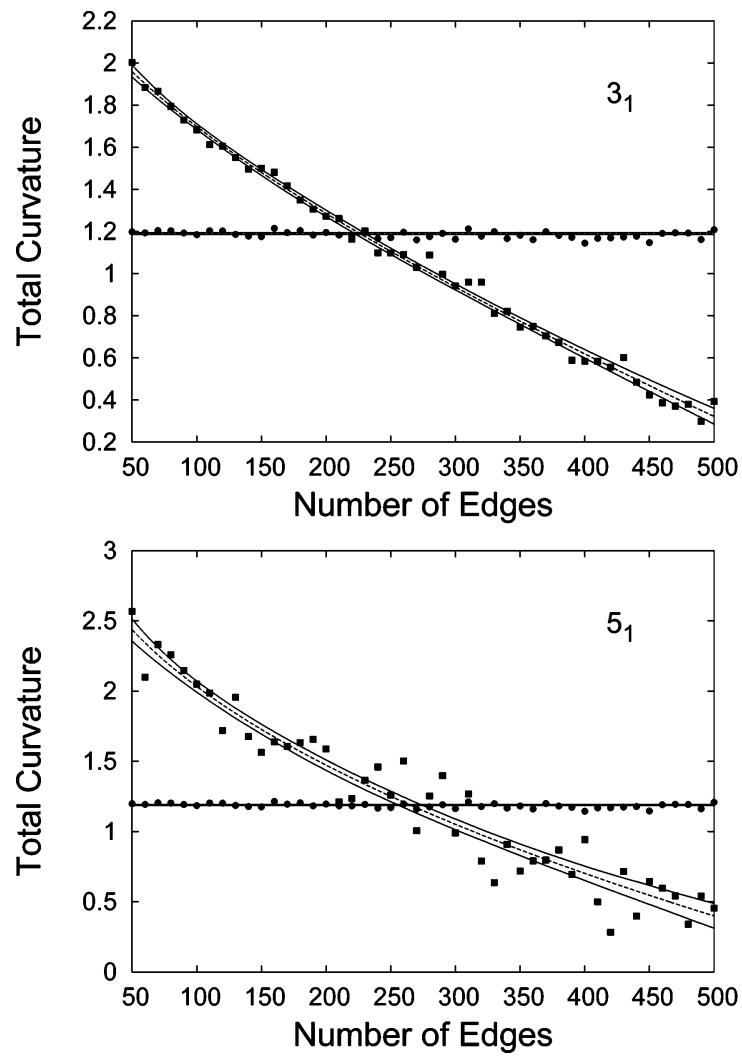

Table 2. Equilibrium Lengths for Total Curvature and Total Torsion and the Maximum Probability Lengths

\begin{tabular}{|c|c|c|c|c|c|c|}
\hline \multirow[b]{2}{*}{ knot } & \multicolumn{2}{|c|}{ total curvature } & \multicolumn{2}{|c|}{ total torsion } & \multicolumn{2}{|c|}{ maximum probability } \\
\hline & $\begin{array}{l}\text { equilib } \\
\text { length }\end{array}$ & \pm & $\begin{array}{l}\text { equilib } \\
\text { length }\end{array}$ & \pm & $\begin{array}{c}\text { max prob } \\
\text { length }\end{array}$ & \pm \\
\hline $3_{1}$ & 226.34 & 4.44 & 229.40 & 2.23 & 235.46 & 2.47 \\
\hline $4_{1}$ & 241.27 & 6.14 & 245.36 & 4.67 & 247.09 & 2.75 \\
\hline $5_{1}$ & 264.85 & 9.60 & 279.00 & 7.94 & 265.53 & 8.05 \\
\hline $5_{2}$ & 275.22 & 7.85 & 276.01 & 5.49 & 261.20 & 5.90 \\
\hline $6_{1}$ & 286.94 & 16.52 & 291.72 & 10.02 & 275.99 & 9.24 \\
\hline $6_{2}$ & 301.06 & 13.90 & 302.56 & 8.93 & 276.21 & 8.12 \\
\hline $6_{3}$ & 289.13 & 18.15 & 307.02 & 10.31 & 284.48 & 7.88 \\
\hline
\end{tabular}

unit sphere and the Mersenne Twister method due to Matsumoto and Nishimura ${ }^{15}$ (which is initialized using gsl_rng_mt19937) as our random number generator. We then add the $n / 2$ edge vectors with opposite directions to our sample. Note that the sum of the now $n$ vectors is the zero vector. To create a polygon from these vectors, one could simply shuffle the order of the vectors and use these as the edge vectors of the polygon. However, this method would constrain us in a low dimensional subspace of the space of all equilateral polygons. This constrained subspace may have rather different properties and statistics as compared to the entire space.
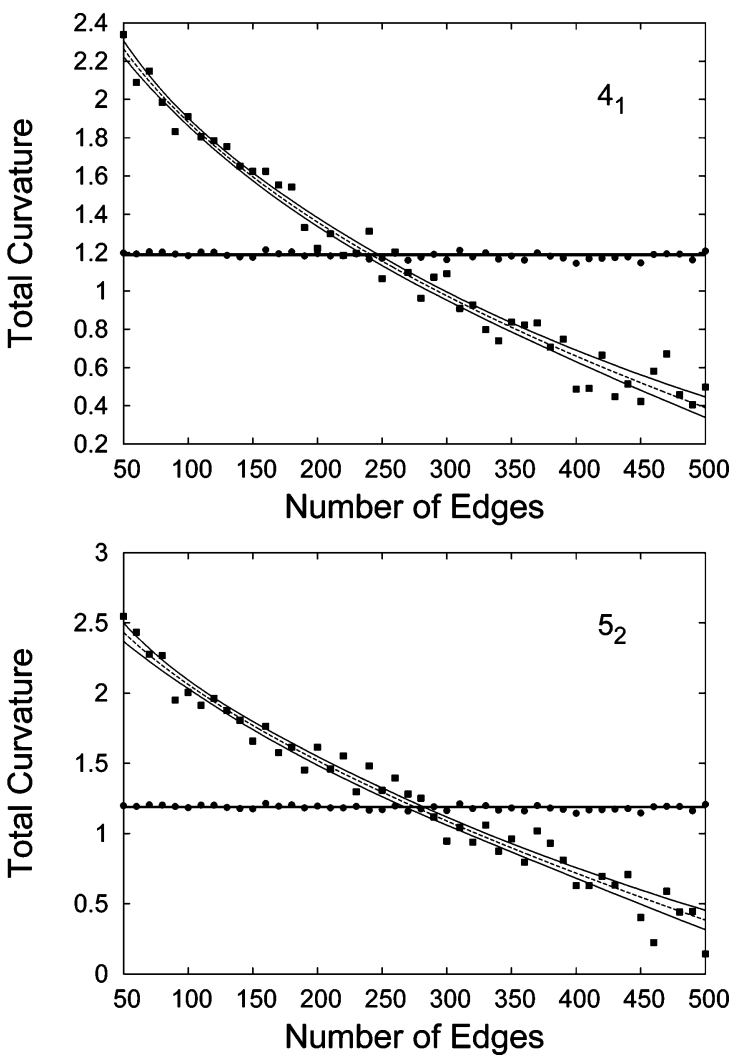

Figure 5. Normalized mean total curvature and summary curves from the MCMC analysis for the three, four, and five crossing knots. 

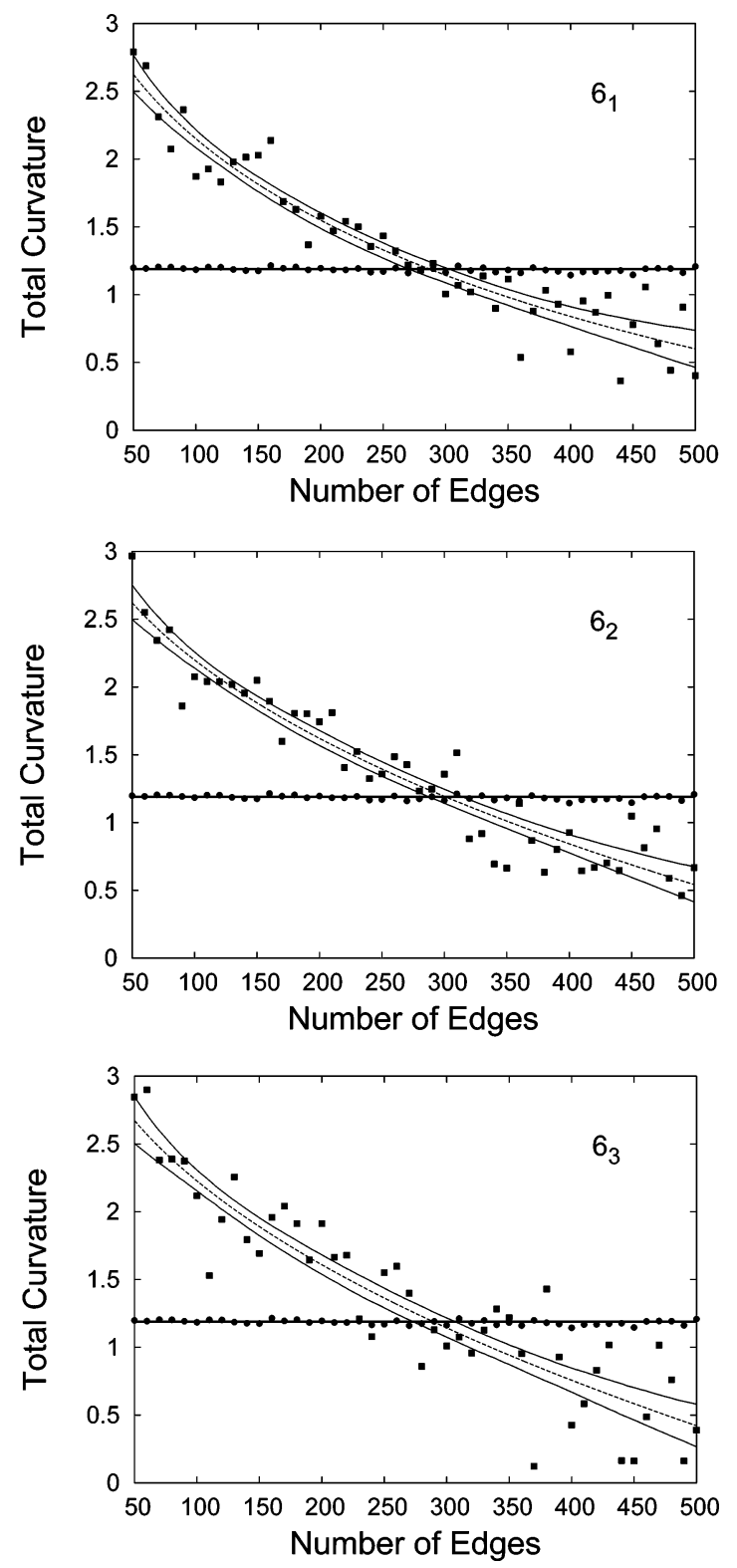

Figure 6. Normalized mean total curvature and summary curves from the MCMC analysis for the six crossing knots.

To accomplish a wider sampling, we use a process to break the correlations between the $n / 2$ pairs of opposite vectors. We choose two vectors, at random, from the sample and replace them with the result of rotating the vectors by a random angle about the axis determined by their sum. We call this procedure a pairwise-crankshaft rotation, abbreviated as PCR. This leaves the sum of the vectors constant but changes the directions of the two implicated vectors. In our computations, we found that $3 n$ random rotations provided a distribution of total curvatures and total torsions that was consistent with those of data sets generated with many more PCRs as well as with that provided by means of other approaches to random equilateral polygon generation. A brief outline of this analysis is found in the next section.

We next determine the knot type of each of the polygons using the HOMFLY polynomial ${ }^{16}$ program of Ewing and Millett. ${ }^{17}$ The HOMFLY polynomial does not determine the knot type per se, so we really determine the distribution of HOMFLY polynomials of the random polygons. However, for the knot types we analyze in this study, the HOMFLY polynomial is an effective surrogate for the knot type. The associated indeter- minacy is due to small classes of knots that will have little effect on the statistics derived from this data. ${ }^{18}$

We then calculate the total curvature and total torsion for each of the random polygons and update the file associated with its HOMFLY polynomial to keep a running list of these quantities. Knot probabilities and average values are then computed for the knots $3_{1}, 4_{1}, 5_{1}, 5_{2}, 6_{1}, 6_{2}$, and $6_{3}$ and for the entire knot population at each number of edges. Because the true average spatial values for right- and left-handed versions of a chiral knot will be the same, we have grouped them together after checking that the differences in their equilibrium lengths were small. These differences are due to statistical fluctuations, providing an independent estimate of the error associated with this method. In order to estimate the error intrinsic to the calculation, we have undertaken a rigorous analysis using a method that will be described in section 5. The computations were completed on a cluster of 39 Pentium-4 $2.8 \mathrm{GHz}$ desktop computers and took $\sim 2$ months of continuous run time.

\section{The Hedgehog Method}

The goal of this section is to describe our implementation of the hedgehog algorithm in which we used 3 times the number of edges worth of pairwise-crankshaft rotations (PCRs) to generate the set of 400000 random polygons. We will show that, in fact, a surprisingly small number of PCRs are sufficient to provide statistically robust data for the total curvature and total torsion. This does not prove, however, that the full space of polygons was sampled but, rather, shows that additional PCRs do not provide statistically significantly different estimates of the total curvature and total torsion.

The probability density function of the total curvature and total torsion distributions for the space random equilateral polygons is unknown. The question, then, was how to determine whether our random polygon generation algorithm provided a robust data set. To do so, we turned to the KolmogorovSmirnov (KS) two-sample test ${ }^{19}$ which tests whether two sets of observations are from the same (but unknown) distribution. The KS test is a measurement on the cumulative distribution functions $(\mathrm{CDF})$ of the sample sets and relies on a value $D_{\mathrm{mn}}$, the maximum height difference between the CDFs of the two sets of observations. When the value of the KS statistic is less than 1.36 , we can conclude, with $95 \%$ confidence, that the observations do not come from different distributions.

We generated 100000 polygons using $n, 2 n, 3 n, 4 n, 5 n$, and $n^{2}$ PCRs for numbers of edges $n$ taking values 100, 200, 300, 400 , and 500 edges. As a first test, we compared the $k n$ PCR samples for both total curvature and total torsion vs the samples using $n^{2}$ PCRs. If there was a difference in using $O(n)$ vs $O\left(n^{2}\right)$ to determine the total curvature and total torsion distributions, then these comparisons should have shown some indication of a difference.

The KS statistic values are shown in Figure 1. Note that all of the KS statistic values are less than 1.36. This shows, at least in the case of total curvature and total torsion, that using $n^{2}$ PCRs was no more effective than using $n$ PCRs in the hedgehog algorithm.

As a second test, we compared the total curvature and total torsion distributions using the hedgehog algorithm vs the distributions from a standard Metropolis Monte Carlo algorithm to generate random polygons. For all of the Monte Carlo polygons, we started from a regular $n$-gon and used random crankshaft rotations to determine a set of polygons. We generated 100000 polygons for the same set of edges. We used $10 n^{2}$ crankshaft rotations to move away from the initial state (a 

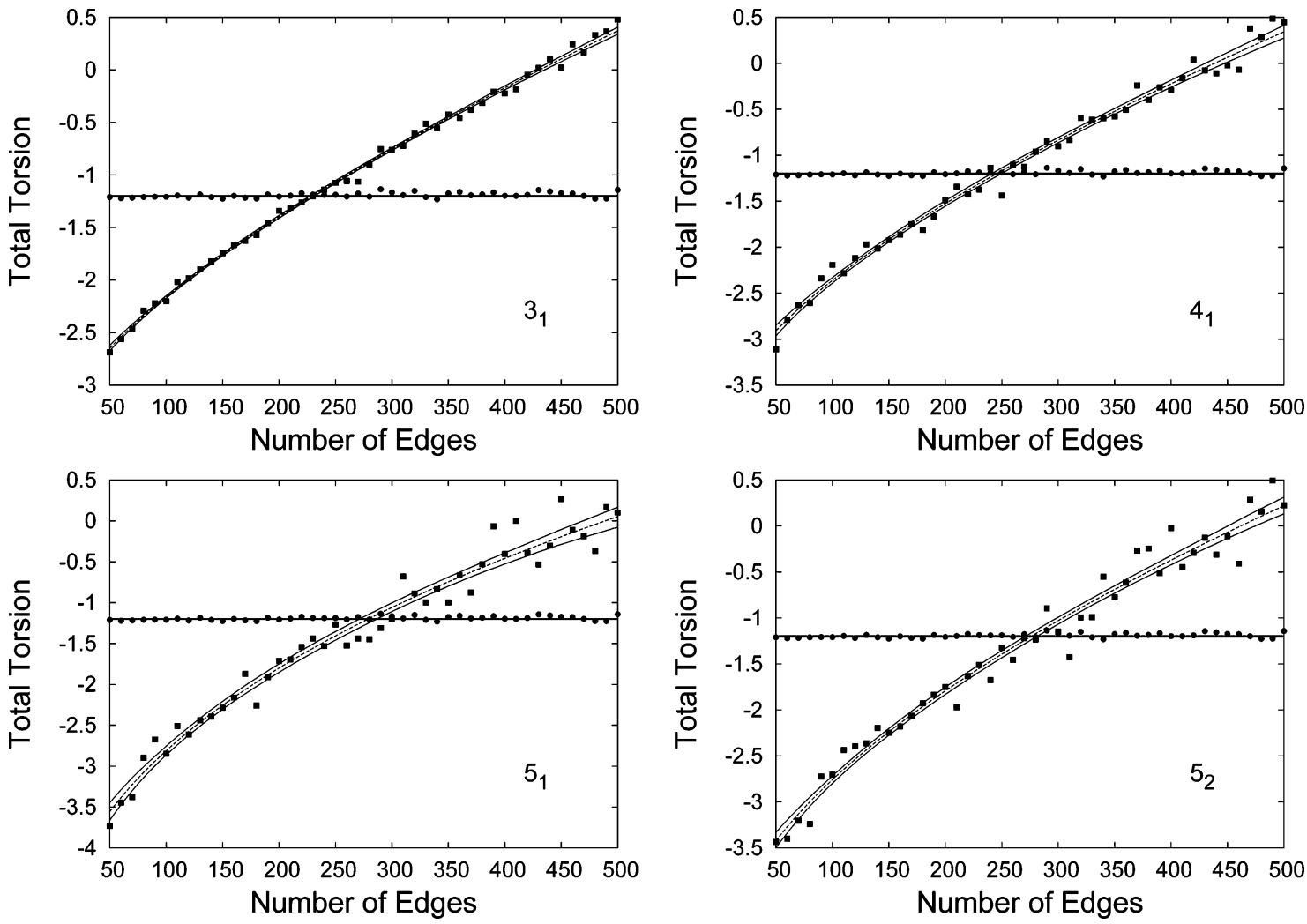

Figure 7. Normalized mean total torsion and summary curves from the MCMC analysis for the three, four, and five crossing knots.

regular $n$-gon) and then $n^{2}$ crankshaft rotations between samples. Again, we used the KS two-sample test. The results are shown in Figure 2. The statistical properties of the Monte Carlo polygon data agree with those of earlier studies. ${ }^{20} \mathrm{We}$ have not attempted to determine the minimal number of random crankshaft rotations required to eliminate the correlations inherent in the initial state since we employ the hedgehog method in this project.

One should expect $\sim 5 \%$ of the KS statistic values to lie above 1.36 when the two samples come from the same distribution, so the two values lying above 1.36 in Figure 2 are not a concern. Overall, we see strong evidence that even $n$ PCRs was enough to generate a robust sampling of the total curvature and total torsion of random polygons.

We have, thus, demonstrated that our implementation of the hedgehog algorithm for generating random equilateral polygons provides a statistically robust data set.

\section{Scaling of Total Curvature and Total Torsion}

An elementary analysis suggests that both the total curvature and total torsion should scale linearly with respect to the number of edges in the polygon. Indeed, upon inspection, the scaling profiles for the knot types and the phantom polygons appear to be linear (see Figure 3 ). In a random walk with $n+2$ vertices (i.e., $n$ freely bending joints), the mean total curvature will be $(\pi / 2) n$ since each joint, on average, will contribute $\pi / 2$ worth of bending. This suggests that the slope of the linear scaling for the two quantities likely has slope $\pi / 2$. To get a sense of the fine structure of this scaling, we subtracted $\pi n / 2$ from the total curvature. These results, shown in Figure 4, clearly exhibit a systematic structure that differentiates between the various distinct knot types.

After this normalization, one sees that the fine structure of the scaling profile of the phantom polygons is constant. For the two quantities, we fit a curve of the form $y=A$ to the normalized scaling profiles of the phantom polygons.
This is equivalent to using a scaling profile of $A+\pi n / 2$ on the original data.

The individual knot types, however, scale differently. In analogy to the work in Orlandini et al., ${ }^{21}$ we employ a linear growth rate for the polygons with fixed topology and multiply by a correction term $A^{\prime}+B / \sqrt{x}+C / x$. In other words, we use a scaling profile of $x\left(A^{\prime}+B / \sqrt{x}+C / x\right)$ where $A^{\prime}, B$, and $C$ are parameters to be fit. After normalizing, we have a scaling function of the form $A x+B \sqrt{x}+C$ where the $A$ value here is the difference of the $A^{\prime}$ above and $\pi / 2$. Because of the similarity of the structure of the data described in the work of Orlandini et al. and that in this research, the proposed fitting function is effective despite the possible differences between self-avoiding polygons and random equilateral polygons. As we have seen earlier, 6,7 there is a similarity between polygons with fixed topology and no excluded volume, they have a scaling exponent $v=0.588$, which is also the scaling exponent of self-avoiding walks attributed to the influence of excluded volume.

Once the scaling has been established, we determine the equilibrium length of the knot types with respect to total curvature and total torsion. We use a Monte Carlo Markov chain model from Bayesian statistics to estimate the likely values of $A, B$, and $C$ for each knot type with respect to each of total curvature and total torsion, to determine the equilibrium lengths, and to determine the likely accuracy of our calculations. This method and our implementation are briefly described in the next section.

\section{Monte Carlo Markov Chain Method}

We describe the algorithm for determining the scaling functions, giving the equilibrium length numbers, and for providing error bars for the normalized data as well as our estimates. The explanation below concerns individual knot types. For the phantom polygons, the fitting function is simply $g(x)$ $=A$ and the algorithm works analogously. 

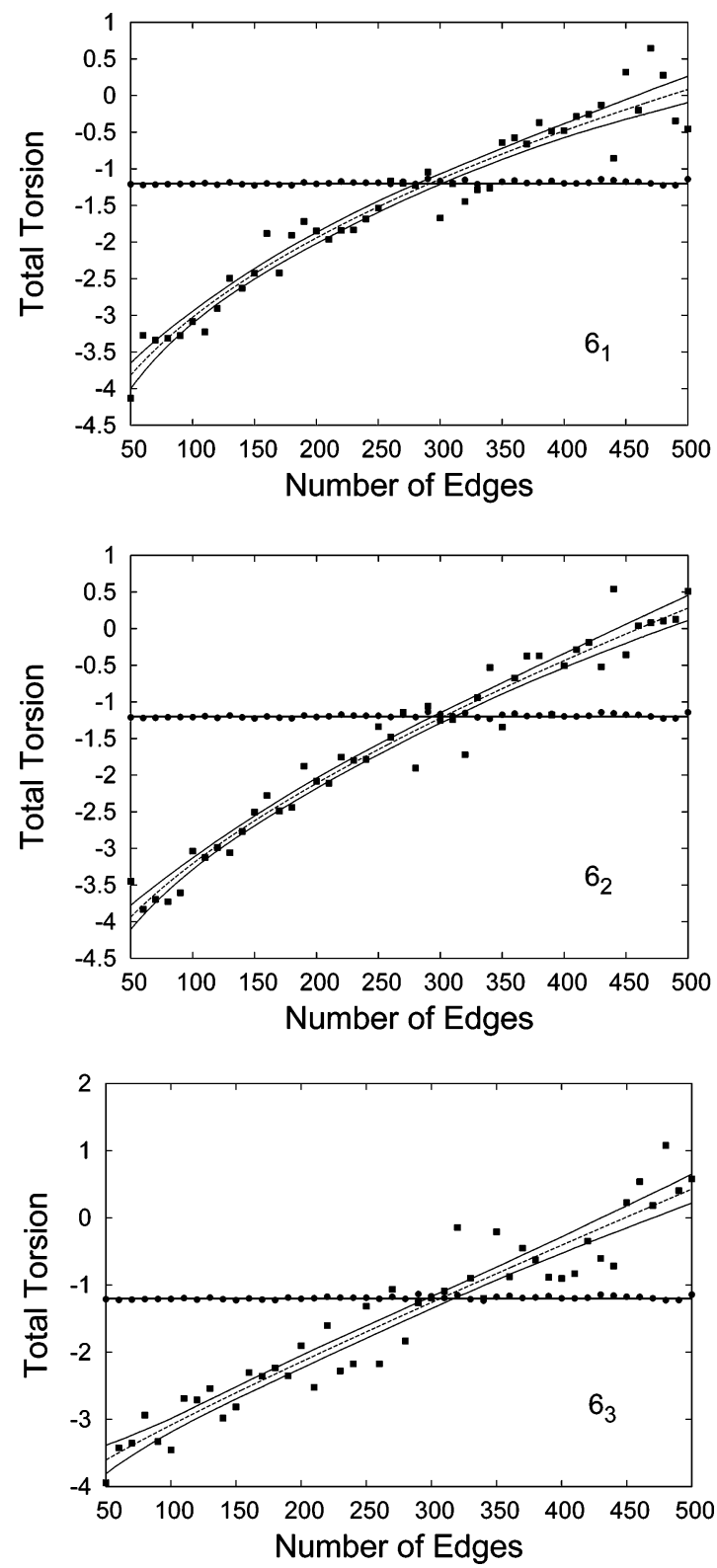

Figure 8. Normalized mean total torsion and summary curves from the MCMC analysis for the six crossing knots.

To estimate the parameters $A, B$, and $C$ of the mean function $g(x)=A+B / \sqrt{x}+C / x$, we implement the Metropolis Markov Chain Monte Carlo (MCMC) sampling method (for more details, see e.g. refs 22 and 23).

A key facet of the analysis is the use, for the knot types, of $g(x)$ (i.e., $(A+B / \sqrt{x}+C / x)$ which comes from ref 21 where it was used for the scaling of the radius of gyration of self-avoiding phantom polygons. The scaling functions for self-avoiding polygons are typically used also for modeling polymers with topological constraints. ${ }^{1}$ For phantom polygons, the squared radius of gyration scales as a linear function as does, visibly, the total curvature and total torsion in the same population. For individual knot types, the scaling exponent is $1.176 .{ }^{6}$ The method begins with initial parameters $A, B$, and $C$. It then uses the algorithm to propose new values of the parameters and, with some probability, accepts the new value and repeats the process. These values can then be used to estimate a " $g$ ", and again repeating the procedure, these " $g$ ' $s$ " are used to produce a final average estimated " $g$ ".

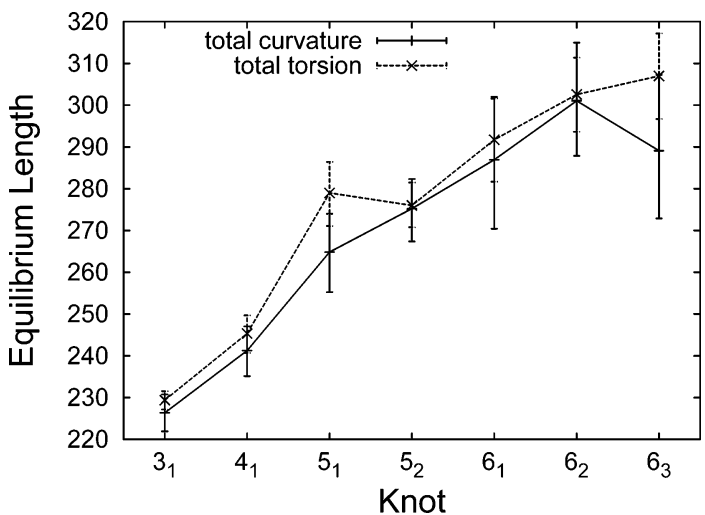

Figure 9. Equilibrium lengths for total curvature and total torsion and their error bars.

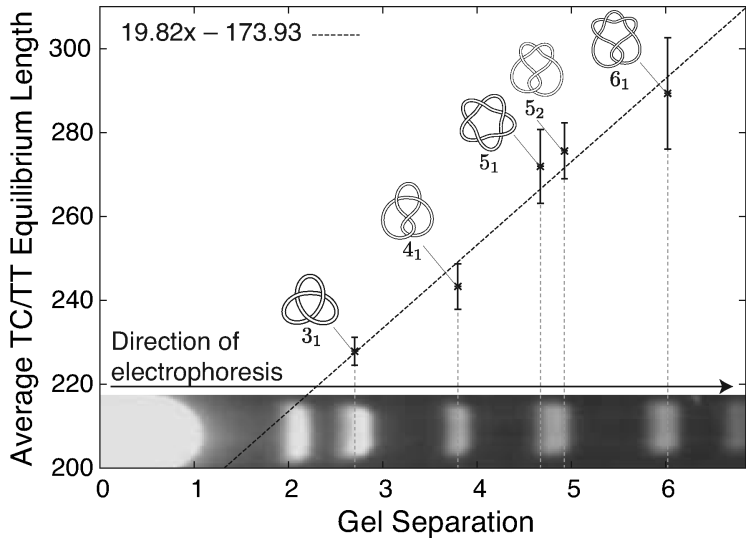

Figure 10. Electrophoretic separation from the position of unknots to specific knot types vs the average total curvature and total torsion equilibrium lengths of the corresponding knot type. The first and second bands are unknotted and linear segments, respectively. The gel image was kindly provided by N. Crisona (UC Berkeley).

We use gnuplot ${ }^{24}$ to determine initial values of $A, B$, and $C$. A delicate aspect of this method was to identify proposal distributions for these parameters which would quickly eliminate the autocorrelation within the sequences of $A, B$, and $C$ values. This was accomplished only after significant ad hoc experimentation in choosing a percent of, for example, $A$ to express the standard deviation of the distribution of proposal values. Ultimately, we found proposal distributions which resulted in lags of 300 (i.e., in all three parameter values losing the autocorrelation in 300 steps of the algorithm). Thus, we required 300 steps of the algorithm to determine one proposed fitting curve. In the end, we have 1000 potential fitting curves for each knot type in addition to the phantom polygons and for each of total curvature and total torsion.

In Figures 5 and 6, we show the summary fitting graphs for each of the knot types with respect to total curvature. In particular, the points are the mean total curvature values at each number of edges as well as for the phantom polygons. The upper, middle, and lower curves are computed as follows. At each number of edges (here we use a step size of one), we compute the value of the fitting graph for each of the 1000 curves. The middle graph is the mean of the 1000 values at each number of edges. We then order those values. The upper and lower curves are the top and bottom values one obtains after removing the largest and smallest $2.5 \%$ of the values. The summary curves give the final prediction for the normalized scaling function with $95 \%$ confidence.

In Figures 7 and 8, we apply an analogous analysis for total torsion. To provide the reader with one set of fitting parameters 


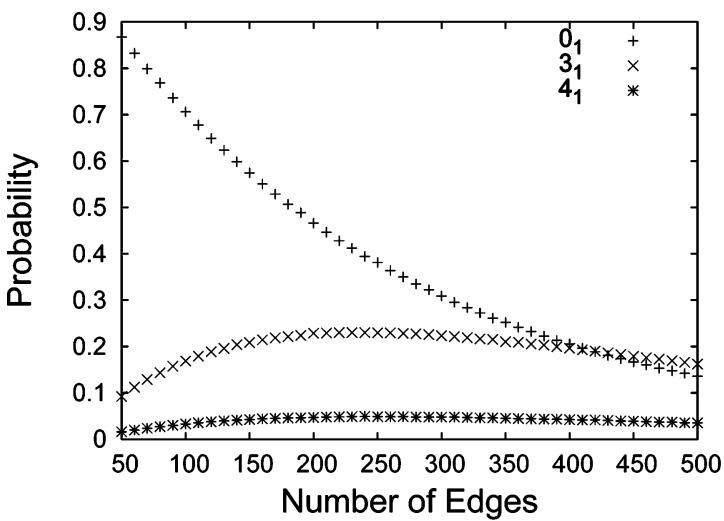

(a)

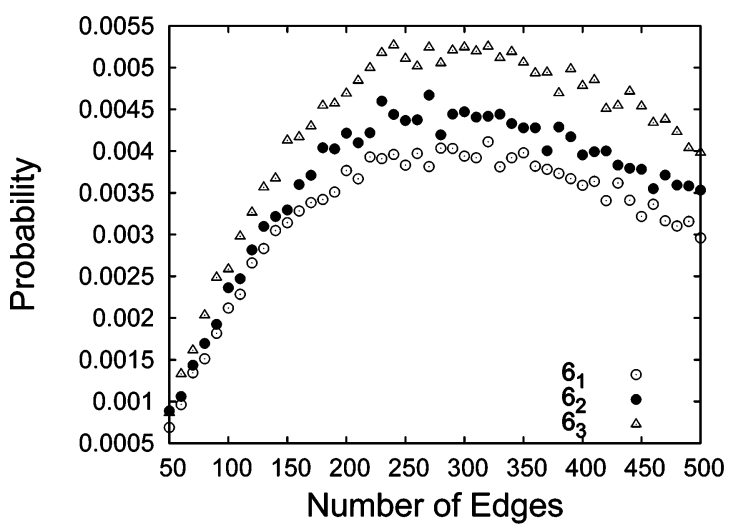

(c)

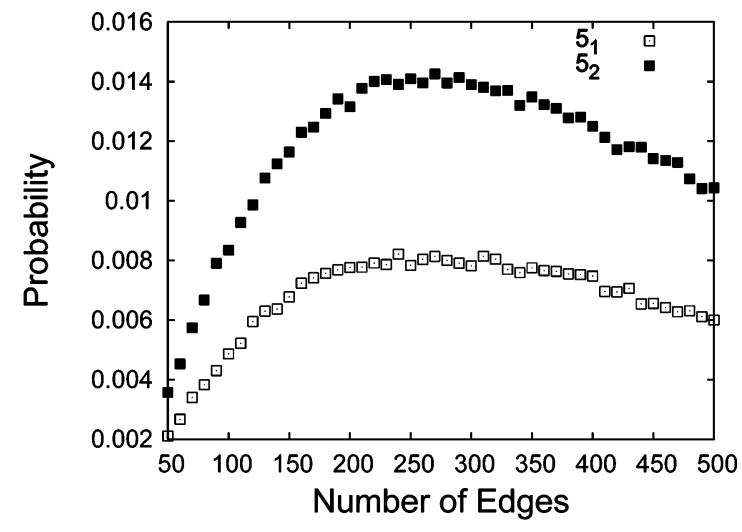

(b)

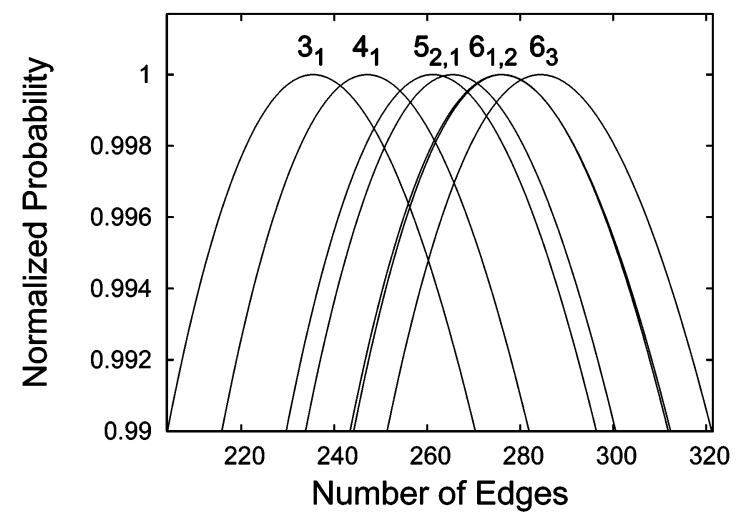

(d)

Figure 11. $(a-c)$ Probability profiles for the analyzed knots. (d) Normalized fitting graphs for the probability profiles.

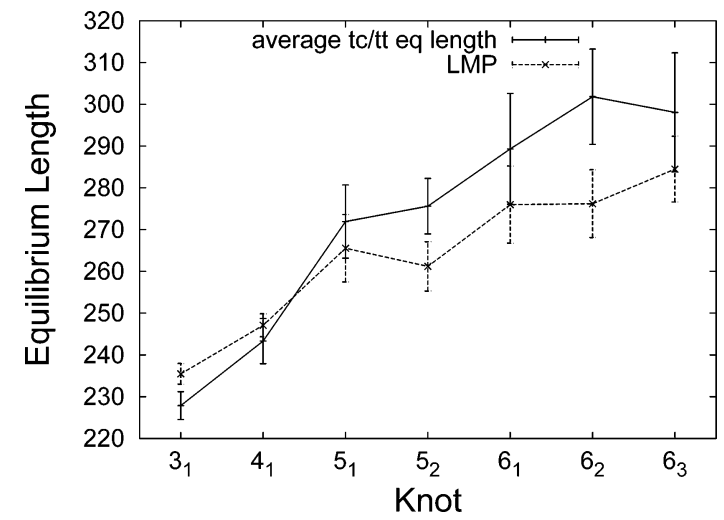

Figure 12. Comparison of the average of the total curvature and total torsion equilibrium lengths and the maximum probability lengths (LMP) for the indicated knot types.

which provide a good fit for the data, we fit a curve of the form $A x+B \sqrt{x}+C$ to the middle graph. These parameter values are reported in Table 1 . They are almost identical to what one gets by averaging the 1000 different $A, B$, and $C$ values.

To determine error bars for the equilibrium length of a given knot type, we computed the crossing point between the potential fitting curves of the knot types and the phantom polygons. Specifically, we computed the crossing point between the first fitting curve for the knot type and the first fitting curve for the phantom polygons. Then we do the same for the second from each list, etc. This process yields a set of 1000 potential crossing points (i.e., equilibrium lengths). We then order the values and remove the first $2.5 \%$ and last $2.5 \%$ of those (i.e., 25 of each) to obtain $95 \%$ confidence intervals for the crossing point (i.e., equilibrium length). We report the mean equilibrium length from this last as well as the error bars for the $95 \%$ confidence interval

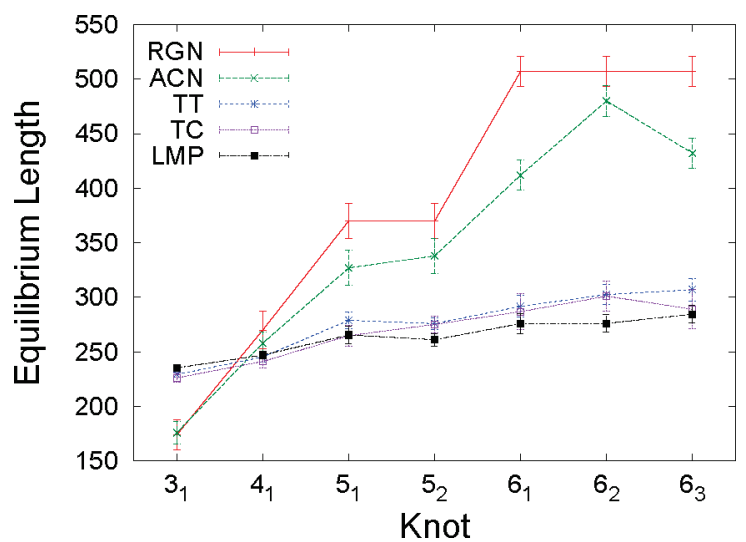

Figure 13. Equilibrium lengths for radius of gyration (RGN), ${ }^{6}$ average crossing number (ACN), ${ }^{7}$ total curvature (TC), total torsion (TT), and the maximum probability lengths (LMP). Note that in ref 6 the six crossing knots were grouped together, so we report the common value.

in Table 2. A graphical rendition of this information is shown in Figure 9.

\section{Analysis}

The calculation of the total curvature and total torsion equilibrium lengths (Table 2) shows that the two values are essentially equal, up to the accuracy of the statistical estimation of their values. As a consequence, these two independent measure of spatial turning and twisting capture, on average, the same characteristics of the spatial equilateral polygons. Together, the total curvature and total torsion equilibrium lengths capture important aspects of the evolution of the average spatial structure of knotted equilateral polygons. As a test of the significance of this evolutionary characteristic, we consider the correlation 
between them and the observed gel separation of the various knot types. Such correlations have already been observed with the average crossing number, but this is the first consideration of the influence of total curvature and total torsion on gel mobility of knotted macromolecules. In Figure 10, one sees evidence of a linear relationship, at least for the simplest knotting of polygons. Since the speed of gel migration of DNA molecules with the same chain length increases with their compaction, ${ }^{25}$ one can conclude that polymers with the same chain length which form different knot types have similar ratios of their overall compaction as the corresponding ratios of the equilibrium lengths for the respective knot types.

An MCMC analysis of the probability profiles of the individual types of knotted polygons, similar to that done for total curvature and total torsion, provides estimates of the lengths at which the different knot types shown in Figure 11 attain their maximum probability. These also correlate with the total curvature/total torsion equilibrium lengths as shown in Figure 12. The total curvature and total torsion are intimately connected to knotting at the geometric level. This correlation suggests that the ratio of the entropy of a given knot to the entropy of all polygons reaches its maximum at the chain length corresponding to the equilibrium length of a given knot with respect to the total curvature or total torsion.

We have observed that the normalized average total curvature and total torsion of the phantom polygons appears to be constant, approximately 1.2 and -1.2 , respectively. A simple estimate derived from the inner product of the sum of the edge vectors ${ }^{26}$ suggests an approximation of 1.0 for the excess total curvature. The case of the total torsion and the search for more accurate estimates remains an interesting research question.

\section{Conclusions}

We have calculated the equilibrium lengths for equilateral random polygons with respect to the total curvature and total torsion of the configurations by showing that the differences in restricted and unrestricted topology already observed for average crossing number and radius of gyration hold for these quantities as well. We note that the total curvature and total torsion equilibrium lengths appear more closely related to the maximum probability lengths than, for example, the average crossing number equilibrium length. The total curvature and total torsion equilibrium lengths are comparable to these and provide both a strong correlation with respect to observed physical properties, e.g., the gel separation, and the length of maximum probability. While both the total curvature and total torsion scale, as predicted, linearly as a function of the number of edges in the configuration, there appears to be an excess/deficit of that same magnitude, 1.2, which has not been entirely explained theoretically and which would be associated with the closure of the polygons.
Acknowledgment. The authors thank John Sullivan and Alexander Y. Grosberg for helpful suggestions. A.D. was supported by SNF Grant \#PA00A-105000. M.P., P.P., and E.R. were supported by NSF Grant \#DMS0311010. A.S. was supported by Swiss National Science Foundation Grants 3152068151 and 3100A0-103962.

\section{References and Notes}

(1) Deutsch, J. M. Phys. Rev. E 1999, 59, 2539.

(2) Grosberg, A. Y. Phys. Rev. Lett. 2000, 85, 3858.

(3) Moore, N. T.; Lua, R. C.; Grosberg, A. Y. Proc. Natl. Acad. Sci. U.S.A. 2004, 101, 13431

(4) Shimamura, M. K.; Deguchi, T. J. Phys. A 2002, 35, 241.

(5) Yao, A.; Tsukahara, H.; Deguchi, T.; Inami, T. J. Phys. A 2004, 37, 7993.

(6) Dobay, A.; Dubochet, J.; Millett, K.; Sottas, P.-E.; Stasiak, A. Proc. Natl. Acad. Sci. U.S.A. 2003, 100, 5611.

(7) Diao, Y.; Dobay, A.; Kusner, R. B.; Millett, K. C.; Stasiak, A. J. Phys. A 2003, 36, 11561.

(8) Millett, K.; Dobay, A.; Stasiak, A. Macromolecules 2005, 38, 601.

(9) de Gennes, P. G. Scaling Concepts in Polymer Physics; Cornell University Press: Ithaca, NY, 1979.

(10) Milnor, J. W. Ann. Math. 1950, 52, 248.

(11) Janse van Rensburg, E. J.; Promislow, S. D. J. Knot Theory Ramifications 1999, 8, 463.

(12) Moore, N. T.; Lua, R. C.; Grosberg, A. Y. In Physical and Numerical Models in Knot Theory; Calvo, J. A., Millett, K. C., Rawdon, E. J., Stasiak, A., Eds.; World Scientific Publishing: Singapore, 2005; Vol. 36, pp 363-384.

(13) Moore, N. T.; Lua, R. C.; Grosberg, A. Y. n Physical and Numerical Models in Knot Theory; Calvo, J. A., Millett, K. C., Rawdon, E. J., Stasiak, A., Eds.; World Scientific Publishing: Singapore, 2005; Vol. 36, pp 385-398.

(14) Klenin, K. V.; Vologodskii, A. V.; Anshelevich, V. V.; Dykhne, A. M.; Frank-Kamenetskii, M. D. J. Biomol. Struct. Dyn. 1988, 5, 1173.

(15) Matsumoto, M.; Nishimura, T. ACM Trans. Model. Comput. Simul. 1998, 8,3 .

(16) Freyd, P.; Yetter, D.; Hoste, J.; Lickorish, W. B. R.; Millett, K.; Ocneanu, A. Bull. Am. Math. Soc. (N.S.) 1985, 12, 239.

(17) Ewing, B.; Millett, K. C. In Progress in Knot Theory and Related Topics; Hermann: Paris, 1997; pp 51-68.

(18) Hoste, J. In Handbook of Knot Theory; Elsevier: Amsterdam, 2005; pp 209-232.

(19) DeGroot, M. H.; Schervish, M. J. Probability and Statistics; Addison Wesley: Reading, MA, 2001; Chapter 9, pp 572-572.

(20) Millett, K. C. In Knots in Hellas '98; World Scientific Publishing: Singapore, 2000; pp 306-334.

(21) Orlandini, E.; Tesi, M. C.; Janse van Rensburg, E. J.; Whittington, S. G. J. Phys. A 1998, 31, 5953 .

(22) Metropolis, N.; Rosenbluth, A.; Rosenbluth, M.; Teller, A.; Teller, E. J. Chem. Phys. 1953, 21, 1087.

(23) Gilks, W. R., Richardson, S., Spiegelhalter, D. J., Eds. Markov Chain Monte Carlo in Practice; Chapman and Hall: London, 1996.

(24) gnuplot, http://www.gnuplot.info, program for plotting and fitting data.

(25) Vologodskii, A. V.; Crisona, N. J.; Laurie, B.; Pieranski, P.; Katritch, V.; Dubochet, J.; Stasiak, A. J. Mol. Biol. 1998, 278, 1.

(26) Grosberg, A. Y. personal communication, 2006.

MA0627673 